27 | 2001

Histoire de l'enseignement du français langue

étrangère ou seconde dans le bassin méditerranéen.

Volume 1

\title{
Aperçu sur l'enseignement du français en Tunisie de 1830 à 1883
}

\section{Abderrazak Bannour}

URL : https://journals.openedition.org/dhfles/2604

DOI : $10.4000 /$ dhfles.2604

ISSN : 2221-4038

Éditeur

Société Internationale pour l'Histoire du Français Langue Étrangère ou Seconde

Édition imprimée

Date de publication : 1 décembre 2001

ISSN : 0992-7654

\section{Référence électronique}

Abderrazak Bannour, « Aperçu sur l'enseignement du français en Tunisie de 1830 à 1883 », Documents pour l'histoire du français langue étrangère ou seconde [En ligne], 27 | 2001, mis en ligne le 31 janvier 2014, consulté le 27 mai 2021. URL : http://journals.openedition.org/dhfles/2604 ; DOI : https:// doi.org/10.4000/dhfles.2604

Ce document a été généré automatiquement le 27 mai 2021.

(c) SIHFLES 


\title{
Aperçu sur l'enseignement du français en Tunisie de 1830 à 1883
}

\author{
Abderrazak Bannour
}

\section{Introduction}

1 Les deux bornes choisies de 1830 à 1883 ne sont nullement arbitraires. La date de 1830 correspond certes à un événement majeur, celui de l'occupation de l'Algérie par la France. La proximité territoriale de l'Algérie a certainement facilité l'accès des Français à la Tunisie. Mais les nouveaux venus, qui ont fondé des établissements d'enseignement, sont tous arrivés, par la mer, de la métropole. En plus, les Français n'ont pas attendu cette date pour s'installer en Tunisie. Car la colonie française y était déjà installée depuis longtemps (le premier consulat a été ouvert le 28 mai 1577, sous Henri III). Certaines grandes familles comme les Chapeliers, Gaspari, Gandolphe, Van Gaver, y étaient depuis le XVI, d'autres depuis le XVII ${ }^{e}$. Mais l'ouverture du premier consulat ne correspond pas à l'afflux des colonies européennes venues s'installer en Tunisie. Chater (1978 : 33-34) pense que les premiers venus en masse, après les démonstrations navales européennes ${ }^{1}$ en Tunisie qui se sont succédé de 1816 à 1819, étaient les Maltais. Ils ont été suivis de près par les Siciliens ${ }^{2}$. Ceux-ci l'ont fait à partir de 1820 .

2 Cette date de 1830 correspond en réalité à la création des premiers établissements d'enseignement privé. Ces établissements n'étaient ni l'œuvre des Tunisiens ni l'œuvre des Français, mais des Italiens, qui formaient le clergé, et des Anglais protestants, au profit des Maltais ${ }^{3}$. Les Tunisiens musulmans avaient spécialement le kouttab (école coranique) à côté des madarsas qui servaient surtout une culture religieuse, mais nullement scientifique ou linguistique. En 1830, la France vivait elle-même une crise relative à l'enseignement. Les Français avaient donc trop à faire en vue de réformer l'enseignement, le laïciser, l'extraire de la domination de l'église ${ }^{4}$ pour penser à étendre ou développer l'enseignement à l'étranger. Comme disait Marcel Gandolphe (1922 : 207) : «Seule la France n'avait pas encore tenté le moindre effort ». Cette date de 1830 
marque en outre la fin du règne de Charles $\mathrm{X}$ et le début de celui de Louis-Philippe ${ }^{5}$, et en Tunisie une très mauvaise saison agricole marquée par une grande sécheresse.

On aurait pu commencer ce travail par la date d'installation, grâce à Sainte Emilie de Vialar ${ }^{6}$, de la première école dans laquelle a été dispensé un enseignement en français, à savoir 1840. Cette action a été sans doute encouragée par la situation politique dans l'empire ottoman finissant. Le régime, dit de capitulation, a favorisé les puissances occidentales qui ont été aux côtés des Ottomans dans leur guerre de Crimée contre la Russie tsariste. Ces facilités exprimées dans les différents édits, par exemple l'édit de Chérif Gulkhana (1839), ont pour but de mettre les sujets européens sous la protection de la France ou de l'Angleterre, de garantir la liberté du culte, de l'enseignement et du commerce. Mais cela nous aurait de toute manière ramené vers cette date clé de 1830 . La création de cette première école française se trouve justifiée par ce qui s'est passé en Tunisie, en France et en Europe une dizaine d'années plus tôt. Ce sera-là une première hypothèse de travail : le but du développement de l'enseignement du français en Tunisie n'était pas de promouvoir la langue française, encore moins de s'occuper de la colonie française en Afrique $^{7}$, mais de s'opposer à l'influence grandissante des concurrents européens (l'Angleterre et surtout l'Italie), et ainsi de préparer l'installation du Protectorat.

Il aurait été plus naturel d'arrêter notre recherche à 1881, date de l'installation du Protectorat (but ultime de l'effort d'implantation du français en Tunisie, d'après la précédente hypothèse de travail). $1881 \mathrm{a}$ vu une chute très nette dans le nombre d'élèves de confession musulmane fréquentant les écoles françaises, très probablement en réaction à l'occupation du pays par l'année française. 1889 est aussi une date très importante. Elle correspond à l'apogée par l'explosion du nombre d'écoles : ce nombre étant passé de 24 en 1883 à 69 en 1889 (voir graphique en lin d'article). Le nombre d'écoles et donc d'élèves aurait triplé en 6 ans ! Mais la date de 1883 est loin d'être arbitraire. Elle correspond à des événements majeurs qui ont à jamais marqué le statut de la langue française en Tunisie. Le premier correspond à la création de la Direction de l'Enseignement Public qui a mis en application les dispositions contenues dans la loi Guizot $^{8}$, sous la direction de Louis Machuel', arabisant d'Alger et ancien inspecteur des écoles libres françaises en Tunisie. Le second correspond à la création de la Ligue Française de l'Enseignement dont l'œuvre d'encouragement à la lecture (par la collecte et les dons de livres) a eu énormément d'impact sur le développement de la langue française en Tunisie. Le dernier, mais pas le moindre, est l'institutionnalisation du bilinguisme par l'édition bilingue ${ }^{10}$, arabe/français, du Journal officiel Tunisien.

\section{Contexte historique}

5 Il transparaît à la lecture des historiens que, par l'établissement des écoles libres françaises, ni la culture ni la langue françaises n'étaient un but en soi, mais un travail préliminaire à l'installation du Protectorat. La Tunisie n'était qu'une partie d'un plan global d'acculturation qui visait sinon l'Afrique du moins le Maghreb. II s'agissait ainsi de contrer l'influence grandissante du protestantisme (écoles financées par les Britanniques et les Américains surtout en Orient et dans les pays sous domination turque), en créant un enseignement catholique. Il faut reconnaître cependant que l'installation de tels établissements catholiques faisait écho à la querelle qui secouait la société française concernant l'enseignement. L'établissement de ces écoles 
congréganistes permettait de limiter l'influence des écoles laïques. Il ne faut donc pas s'étonner de voir que toutes les écoles fondées en Tunisie avant 1883 étaient le fait de congréganistes. Mais, au sein même de l'église catholique, il était vital de devancer les Italiens (du royaume de Sardaigne), en Tunisie et en Afrique du Nord, en instituant sinon une culture française et une reconnaissance culturelle dans le français, du moins des liens affectifs avec la France. Les lettres pastorales du cardinal Charles Lavigerie confirment bien qu'il s'agissait d'une réalité et non de la projection d'un scénario possible. Lavigerie lui-même était l'un des acteurs principaux de cette manœuvre. Marcel Gandolphe (1922 : 209-210.), relatant les conditions d'installation des institutions d'enseignement dans la Régence, atteste indirectement cette course à la présence culturelle contre l'Italie :

En 1880, M. Barthélémy Saint-Hilaire impute sur le budget français la somme de 6000 francs pour l'extension de la langue française. Les Frères des Écoles chrétiennes touchent intégralement cette somme. Le gouvernement italien fut la cause directe de cette mesure bienveillante ; il avait augmenté, en effet, de 6000 francs la subvention accordée aux écoles italiennes.

Ce seraient là les circonstances qui ont aidé à l'installation des institutions d'enseignement du français. Nul ne peut prétendre que ces missions avaient pour but uniquement de donner une instruction "à la française " à la colonie installée en Tunisie. Car, ce n'est justement pas aux Français que cet enseignement a profité. Le pourcentage des Français, comparé à celui des autochtones (juifs et musulmans) et des non-Français (Maltais, Italiens et Espagnols), ainsi que les autres nationalités panni les minorités installées en Tunisie à cette époque, était insignifiant. Ce déséquilibre, qui a duré jusqu'en 1883 et même au-delà, vient confirmer cette hypothèse. Ainsi par exemple, en 1840, l'école d'Emilie de Vialar comptait 25 élèves, parmi lesquelles 15 Italiennes et 10 Maltaises. En 1880, elle comptait 165 élèves dont 86 Italiennes, 73 Maltaises, 1 Française et 5 Israélites. Le nombre d'Italiennes dans cette école s'explique à la fois par l'importance de la colonie et par le fait que la langue italienne faisait partie de la base de l'enseignement dispensé dans cet établissement. En 1865, selon Marcel Gandolphe (1922 : 209), il y avait chez les Frères des Écoles Chrétiennes 287 élèves dont 27 Français, contre 118 Maltais, 81 Italiens, 46 Juifs et Musulmans, 9 Schismatiques (sans indiquer la nationalité) et 2 Grecs. Selon Louis Machuel (1889 : 9), en 1883, on comptait en Tunisie « 150 élèves indigènes seulement étudiant la langue française ; en 1885 , il y en avait 474 , en 1889 , nous en trouvons 1765 ». Selon la même source, la proportion des élèves européens fréquentant les écoles françaises était tombée en 1889 à seulement $9,5 \%$.

\section{Statut de la langue française}

7 En se fondant sur ces statistiques, qui dénoncent la réalité de l'enseignement du français en Tunisie avant 1883, on peut affirmer sans risque d'erreur que bien que les programmes aient repris ceux de la métropole, la langue française était enseignée surtout comme langue étrangère, rarement comme langue maternelle. Il faudrait attendre 1883, pour que son enseignement de par son statut, du moins en théorie, comme langue officielle de la Régence, se complexifie pour se noyer dans l'ambiguïté entre langue maternelle, langue étrangère et/ou langue seconde. 
8 Le français a donc eu un statut principalement de langue étrangère jusqu'en 1883, puis il a eu tendance, dans certains cas, à devenir une langue seconde à partir de cette date. Témoins de ce changement de statut, le nombre d'Italiens et de Tunisiens écrivant en français, promu langue de la littérature et des communications. La langue française était devenue aussi langue des journaux (co-existence en fait avec l'arabe, l'italien et l'hébreu), de l'administration et de la littérature"11. La Tunisie, à partir de 1883, était devenue un pays ayant un superstrat : le français, avec en parallèle des langues communautaires comme l'italien, l'arabe, l'hébreu ou le maltais.

D'après les sources auxquelles nous avons pu accéder directement ou indirectement ${ }^{12}$, l'influence des associations de propagation de la foi apparaissait clairement. Ces Associations étaient chrétiennes, comme par exemple, celles de l'abbé Bourgade, des chapelains de Saint-Louis de Carthage, des Frères des Écoles Chrétiennes, des Sœurs de Saint-Joseph et des Sœurs de Sion. Elles étaient aussi juives, comme par exemple, la London Jews Society et l'Alliance Israélite. Il s'agissait donc de l'exportation de l'enseignement religieux. Cela s'expliquerait, nous semble-t-il, par le fait qu'en France l'église avait perdu le monopole de l'enseignement. En revanche, il lui restait la possibilité de rattraper, de l'autre côté de la Méditerranée, le terrain qu'elle avait perdu en France. Cela ne relevait donc nullement du hasard si les documents d'archives indiquaient la confession des maîtres (chrétiens, laïques, musulmans, juifs, voire schismatiques, etc.) et ceux des élèves.

10 Ce qui précède constitue notre deuxième hypothèse. Nous la reformulons sous forme de question rhétorique : l'enseignement en Tunisie avant 1881, exclusivement congréganiste, se serait-il développé avec le même dynamisme si l'enseignement religieux n'avait pas subi, en France, les restrictions qu'il avait connues au début du $\mathrm{XIX}^{\mathrm{e}}$ siècle?

11 Mais, remarquable est l'absence des jésuites...car, si tout le monde connaît leur apport dans la propagation de la foi chrétienne par l'enseignement, ce qui est étonnant, c'est qu'ils n'aient rien tenté en Tunisie, alors que les enseignants comme les programmes ont très probablement profité de leurs méthodes pédagogiques, surtout dans les lycées secondaires. (Cf. Mediha Moucharrafieh, 1990 : 32-36).

12 Mais, ces différentes congrégations ne seraient pas venues s'installer et enseigner en Tunisie s'il n'y avait pas eu de colonies importantes de chrétiens ou du moins d'Européens ${ }^{13}$. En tout cas, lorsque Emilie de Vialar a débarqué en Tunisie pour fonder sa première école en 1840, il y avait environ 10.000 Européens rien que dans le GrandTunis.

13 Il nous semble donc que les deux facteurs cités plus haut, i.e. la volonté de " pénétration intellectuelle » et "l'exportation de l'enseignement religieux ", se soient conjugués dans un mouvement harmonieux qui a rendu possible l'établissement d'autant d'écoles françaises en Tunisie.

\section{Remarques}

14 Il était tout de même étrange que l'italien, qui était la langue dominante par le nombre de locuteurs, mais aussi à cause d'une tradition séculaire (au Maghreb et au MoyenOrient) sous la forme simplifiée de Lingua Franca, ce sabir auquel les populations méditerranéennes étaient habituées, se fasse doubler aussi facilement par le français. 
Cela est d'autant plus étrange que les ecclésiastiques relevant des différentes institutions cléricales de Tunisie (surtout dans l'archevêché de Tunis) étaient tous des capucins italiens ${ }^{14} \ldots$ et que la colonie la plus nombreuse (après les Italiens) était constituée de Maltais catholiques, exerçant leur rituel dans les églises italiennes, quoique appartenant de par les faits à l'Angleterre ${ }^{15}$. Cela contraste avec la colonie française, qui était moins nombreuse. Mais, au vu de ce qui s'est produit, elle devait être bien plus influente, disposant sans doute de plus de moyens ou de liberté d'action.

Dans les écoles françaises, les langues italienne et arabe n'auraient servi à rien d'autre qu'à l'apprentissage de la lecture ${ }^{16}$. Pourtant, le nombre d'Italiens dans ces écoles françaises n'a cessé d'augmenter. La cause semble avoir été la qualité de l'enseignement prodigué. A la Goulette, par exemple, pourtant appelée "Petite Sicile », puisque plus des trois quarts des étrangers étaient des Italiens, les Frères de Saint-Joseph ont pu fonder en 1855 une école française qui a eu beaucoup de succès. Il est certes vrai que les écoles françaises étaient plus prisées parce que les Sœurs et les Frères se consacraient entièrement à l'enseignement, alors que les maîtres italiens avaient d'autres préoccupations politiques et/ou quotidiennes. Il faut signaler que l'Italie était accaparée à l'époque par des problèmes internes d'unité et d'identité. Ce fait semble avoir eu pour conséquence que la France a été la première parmi les pays européens à bénéficier du Régime des Capitulations accordé par les Ottomans aux pays européens. Elle en a tiré beaucoup d'avantages, sur les plans matériel et moral, d'autant plus que la Sublime Porte lui avait accordé le privilège de veiller aux intérêts des colonies chrétiennes catholiques (même non françaises) où qu'elles se trouvent dans les pays sous domination turque. Ces privilèges, la France les a parfois partagés avec l'Angleterre. Elles étaient toutes deux les alliés des Ottomans dans leur guerre contre la Russie des Tsars. Cela semble suffire à expliquer un autre fait curieux, mais remarquable, quant au statut de la langue française de l'époque. L'école créée en 1878 par l'Alliance Israélite à Tunis a adopté la langue française comme langue d'enseignement. Pourtant les Juifs, mis à part les autochtones, étaient en majorité d'origine italienne ou espagnole. Il semble que lesdits privilèges dont avait joui la France auprès de la Sublime Porte justifient pleinement cet état de fait. Il était donc normal que les Juifs livournais par exemple arborent le pavillon français, ce qui les a fait appeler parfois les «Juifs Francs. » Le Pacte Fondamental ('Ahd al-'amen), signé par Mohamed Bey en 1857 sous la pression de la Sublime Porte, n'a fait que légitimer une réalité sur le terrain ${ }^{17}$.

\section{Conclusion}

Cette mise au point concernant quelques aspects de l'enseignement du français en Tunisie a mis l'accent sur deux points : d'abord, les conditions historiques qui ont favorisé la fondation des établissements d'enseignement, ensuite, le statut qu'avait la langue française dans l'enseignement prodigué. Le deuxième point peut être perçu comme le corollaire du premier.

17 Certains historiens pensent que les efforts qui ont abouti à l'établissement des institutions d'enseignement $d u$ français avaient pour but inavoué la création des conditions favorables à l'installation du Protectorat. Cependant, comme nous le dit Arnoulet (1954: 140-141) : 
Il est curieux de constater que tout ce mouvement qui débuta dès 1830 s'est accompli sans l'intervention des Consuls des différentes nations représentées à Tunis et que ce furent plutôt leurs ressortissants qui favorisèrent le développement des écoles.

Ainsi, ce seraient les administrés, qui, ayant des enfants qu'ils désiraient faire instruire, auraient songé à créer les établissements scolaires. Il est vrai que le gouvernement français n'a administré directement aucun établissement, avant 1883.11 a quand même subventionné ou protégé quelques écoles françaises (Foncin, 1882 : 417). En revanche, le gouvernement italien a administré officiellement les écoles italiennes.

En tout état de cause, que ce mouvement ait été inspiré par les gouvernements ou qu'il ne l'ait pas été, l'enseignement faisait bien l'objet de la lutte d'influence qui s'exerçait dans la Régence entre l'Italie et la France au milieu du XIX ${ }^{\mathrm{e}}$ siècle.

Pourtant, les Italiens et avec eux les Maltais étaient les principaux bénéficiaires des écoles françaises. Ainsi, du fait que l'enseignement de cette langue n'était pas destiné aux petits Français, qui constituaient avant le Protectorat un pourcentage négligeable, comparé à celui des Italiens ou des Maltais, les programmes comme les méthodes avaient dû être adaptés à leurs destinataires. Mais nous n'avons aucun document ${ }^{18}$ pour trancher avec certitude le statut qu'avait la langue française à l'époque que couvre notre recherche. La seule certitude qu'on puisse avoir est qu'elle a été considérée comme un superstrat. Elle ne semble avoir accédé au statut de langue commune, langue de l'administration ou de la littérature qu'à partir de 1889.

\section{Graphe représentant l'évolution du nombre des écoles françaises} de 1840 à 1883 (et 1889)

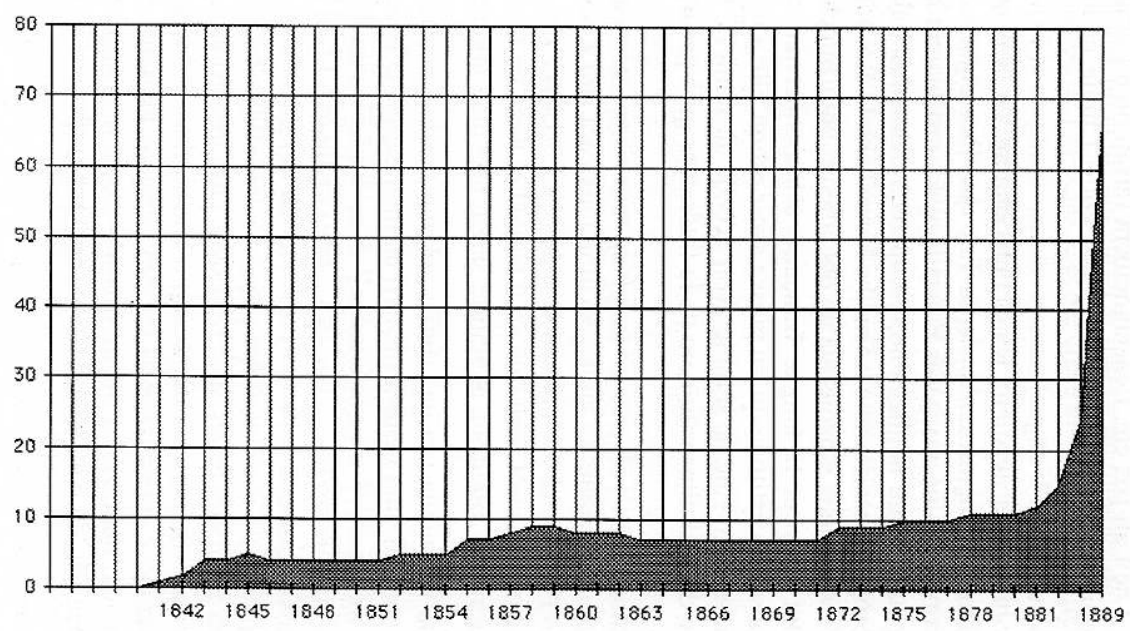

Sources : Les données ont été recueillies dans les différents documents cités en bibliographie. 


\section{Chronologie:}

\section{0}

Occupation de l'Algérie.

Fin du règne de Charles X (1824-1830). Avènement de Louis-Philippe (1830-1848).

Règne de Hassine Bey (1824-1835).

Fondation de la première école protestante par les Anglais au profit des Maltais.

1830/1831, ouverture de la première école israélite (London Jews Society), grâce à Pompeo Sulema, émigré politique livournais, avec le concours de sa sœur Esther.

1835

Avènement de Mustapha Bey.

1837

Avènement de Ahmed Bey (oct. 1837-juin 1855). C'est sous son règne que la plupart des écoles seront installées.

1840

Au cours de l'été, arrive à Tunis la mère Emilie de Vialar, fondatrice des Sœurs de SaintJoseph de l'Apparition, accompagnée de cinq sœurs. Elle ouvre une école gratuite de filles, rue Sidi Saber à Tunis. Elle débute avec 25 élèves : 15 Italiennes et 10 Maltaises.

Morpurgo, aidé de Salone et Luisada, ouvre une école spécialement destinée aux Israélites.

Le 5 mars, Ahmed Bey organise la première école militaire, connue sous le nom de « École polytechnique du Bardo ». Le français était l'une des langues d'enseignement, parce que la documentation et la plupart des manuels étaient rédigés en français.Emilie de Vialar ouvre une école payante (accolée à la première), avec internat et externat pour les filles.

À Sousse, elle ouvre une école de filles, gratuite.

1842

Émilie de Vialar ouvre une école payante (accolée à la première), avec internat et externat pour filles.

À Sousse, elle ouvre une école de files, gratuite.

1843

Emilie de Vialar ouvre l'école de la Marsa qui a été créée sur l'instigation de M. Raffo, ministre du Bey, pour accueillir les fils des notables et les fils des étrangers en poste à Tunis. Cette école n'a fonctionné que pendant deux ans.

1845

L'abbé Bourgade, qui avait « compris toute l'importance qu'il y avait à répandre la connaissance de la langue française dans la Régence » installa son collège dans une impasse de la rue Sidi-el-Mordjani, dénommée de nos jours Zanguet-el-Babbas (impasse du missionnaire). Cet établissement était fréquenté par des enfants de toutes les nationalités et de toutes les confessions. Le programme comprenait l'étude de la langue française et de la langue italienne, des mathématiques, de l'histoire et de la géographie. 
L'établissement du père Bourgade était une école primaire et une école secondaire. Il n'a pas tardé à être uniquement une école primaire. trouva 6 écoles fonctionnelles, 5 écoles de filles fondées par Emilie de Vialar et une école de garçons rue Sidi Morjani à Tunis, fondée par le père Bourgade en 1845, dite Lycée Saint-Louis. a première école fondée à Tunis pendant le règne de Mohamed Bey (le 22 septembre 1855), est la deuxième école de garçons de la Régence. Elle est l'œuvre des Frères des Écoles Chrétiennes. (Soixante élèves furent groupés en deux classes : l'une porta le nom de section française, l'autre de section italienne).

1857

Le Pacte Fondamental donne aux étrangers le droit de posséder des biens immeubles. Ce droit est confirmé par le traité anglo-tunisien de 1863.

1858

Les Frères des Écoles Chrétiennes ouvrent une seconde école rue de l'Église.

1859

Avènement de Mohamed-Sadok Bey (1859-1882).

1863

Le collège du père Bourgade est fermé. Les Frères des Écoles Chrétiennes succèdent à l'abbé Bourgade.

1864

En mars, révoltes du Sahel et de Ben Ghedahem. Elles portent une sérieuse atteinte au nombre d'élèves (une cinquantaine). Les familles, dans la crainte des émeutes, gardent leurs enfants chez elles.

1865

Le calme étant revenu, 287 élèves fréquentent l'école de la rue de la Casbah et de l'Église.

Sur l'ensemble, les nationalités se répartissaient ainsi : 27 Français, 118 Maltais, 81 Italiens. 46 Juifs et Musulmans, 9 Schismatiques [ ? ?], 2 Grecs.

\section{7}

Épidémie de choléra.

1868

Épidémie de typhus (ces épidémies réduisent le nombre des élèves). 


\section{Ali dont ledit collège port
une école franco-arabe.}

1870

1871

1876

1878 dans le même immeuble.

1880 suite collège Saint-Charles. 1881

1882 Charles de Gaulle) à Tunis. d'Afrique) à la Marsa. Saint-Joseph.

III République (1870-1940) [à régime laïc].

Les Frères des Écoles Chrétiennes créent l'école de la Goulette.

« En 1876, le Gouvernement tunisien fonda le collège Sadiki [du nom de Sadok Bey], destiné aux enfants musulmans. Au début, les langues enseignées étaient l'arabe pour tous, les deux tiers des élèves apprenaient soit le français soit l'italien. En 1889, le français était devenu obligatoire. »

L’Alliance israélite ouvre une grande école de garçons. Une école de filles est installée

L’Alliance israélite fonde à Sousse une école de garçons.

« En 1880, le cardinal Lavigerie fit élever, autour de la chapelle Saint-Louis à Carthage, de grands bâtiments dans lesquels il installa un collège qu'il appela collège Saint-Louis de Carthage. Les élèves ne tardèrent pas à affluer ». Ce collège a été rebaptisé par la

En 1880, l'école rue Sidi Saber compte 165 élèves, dont 86 Italiennes, 73 Maltaises, 1 Française, 2 musulmanes et 5 Israélites.

Le lycée Saint-Louis a cédé la place à l'Institut Catholique des Nègres de la Marsa.

Établissement du Protectorat Français en Tunisie. Signature du traité du Bardo.

« En 1881, la population européenne en Tunisie est estimée à 18.914 personnes, dont 708 Français, 11.206 Italiens et 7.000 Maltais (source : Résidence Générale de la République Française, Notice générale sur la Tunisie (1881-1921)).

L'inauguration du collège Alaoui eut lieu le 29 décembre 1881 en présence de S.A. le Bey En novembre 1881, une école libre « laïque », dite l'« institution Morel », est ouverte dans une maison voisine de la douane.

Ouverture le 1er mars de la première école des Sœurs de Sion, rue d'Italie (actuelle rue

Les Marianistes (aidés par Lavigerie) ouvrent une école de garçons à Sousse.

30 octobre, ouverture de l'école des Sœurs Blanches (alias missionnaires de Notre-Dame

2 1er décembre, ouverture d'une nouvelle école à Bizerte par les soins des Sœurs de

4 décembre, les Marianistes ouvrent une école de 3 classes à Sfax.

Décembre, ouverture, par les Sœurs de Saint-Joseph, d'une école de filles à la Cité des Maltais à Tunis. 


\section{BIBLIOGRAPHIE}

\section{Sources manuscrites}

Archives Des Frères Blancs (cartons B4, B5, B5 bis.), des Marianistes (cartons 87, 159, 161), de la République Tunisienne (carton 64, armoire 6 ; carton 71, armoire 7 ; carton 110, armoire 11 ; carton 114, armoire 22), Correspondances de Lavigerie avec Ratisbonne (Bl. 775-776).

\section{Sources imprimées}

ANONYME (1922) : « Le collège Alaoui », La vie Tunisienne Illustrée, avril, 11, 22-24. Tunis, Frédéric Weber. ARNOULET, F. (1954) : « La pénétration intellectuelle en Tunisie avant le Protectorat ». Revue Africaine, vol. XCVIII, 438/439, 140-182. Alger, Société Historique Algérienne.

ARNOULET, F. (1991) : « Les problèmes de l'enseignement au début du Protectorat français en Tunisie (1881-1900) », IB LA, 167, 31-62. Tunis, Revue de l'Institut des Belles Lettres Arabes. AUMONT, Gérard (1939) : «L'œuvre des Sœurs de Saint-Joseph de l'Apparition en Tunisie », Tunisie, 97, 4-7. Tunis, Imprimerie Générale.

BACHROUCH, Taoufik (1985) : «Co-instruction et géographie de la scolarisation primaire en Tunisie (1883-1909) ». Cahiers de Tunisie, T. XXXIII, 133/134, 71-112. Tunis, Faculté des Sciences Humaines et Sociales.

BOURGADE, L'abbé (1852) : Soirée de Carthage, 2e édition. Paris, Jacques Lecoffre. 
CALENDRIER POUR 1881 : Historique de l'Enseignement et des Institutions de la France. Paris, Bray et Rétaux.

CHATER, Khalifa (1978) : Insurrection et répression dans la Tunisie du XIXe siècle. La Mehalla de Zarrouk au Sahel (1864). Publications de l'Université de Tunis.

DESTOURNELLES, De Constant (1891) : La politique française en Tunisie de 1854 à 1891. Paris, Librairie Pion.

DUNANT, Henry (1858) : Notice sur la Régence de Tunis. Imprimerie J. Guillaume Fick, Genève.

FONCIN, Pierre (1882) : «L'enseignement en Tunisie », Revue Internationale de l'Enseignement, T. IV, juillet-décembre, 401-418. Paris, G. Masson.

GANDOLPHE, Marcel (1907) : «L'Abbé Bourgade 1806-1866 ». TAN1T, Revue Tunisienne de Lettres et d'Arts. 1, 106-116. Tunis, Frédéric Weber.

GANDOLPHE, Marcel (1922) : « M. Maccio, consul général d'Italie, avant l'occupation », La vie Tunisienne Illustrée, 11, 23-24. Tunis, Frédéric Weber.

GANDOLPHE, Marcel (1922) : «L'Enseignement français à Tunis avant l'occupation française ». La vie Tunisienne Illustrée, (première partie) 11, 207-210. Tunis, Frédéric Weber.

GANDOLPHE, Marcel (1923) : «L'Enseignement français à Tunis avant l'occupation française ». La vie Tunisienne Illustrée, (deuxième partie) 12, 241-242. Tunis, Frédéric Weber.

GANDOLPHE, Marcel (1955) : «L'enseignement français en Tunisie avant l'occupation française, par les Frères des Écoles Chrétiennes » [extrait du bulletin no 3 du 12 décembre 1923]. Bulletin de l'Association Amicale des Frères des Écoles Chrétiennes de Tunisie, 23, 54-62. Tunis.

GARNIAGE, Jean (1955) : « Les Européens en Tunisie au milieu du 19e siècle (1810-1870) », Les Cahiers de Tunisie, 11, 388-421. Tunis, Institut des Hautes Études.

GRANCHAMP, Pierre (1942) : « Notables français à Tunis de 1592 à 1881 », La Revue Tunisienne, 51, 201-241. Tunis, Institut de Carthage.

IBN ABI DHIEF, Ahmed, (1862-1873) [1971] : (en arabe) Ithaf Ahl az-Zamen bi Akhbar Moulouk Tounis wa Ahd al-Amen. Vol. VI : chronique du règne d'Ahmed Bey (texte établi par A. Abdessalem). Publications de l'Université de Tunis.

JOURNAL OFFICIEL (de Tunisie), de 1883 à 1886.

KRAÏEM, Mustapha (1973) : La Tunisie précoloniale. 2 vol. Tunis, Société Tunisienne de Diffusion.

MACHUEL, Pierre Louis (1889) : L'enseignement public dans la Régence de Tunis. Paris, Imprimerie Nationale.

MACHUEL, Pierre Louis (1906) : L'enseignement public en Tunisie 1883-1906. Tunis, Imprimerie Rapide.

MAHJOUBI, Ali (1977) : L'établissement du protectorat français en Tunisie. Publications de l'Université de Tunis.

MELON, Paul (1884) : « Rapport des Écoles Libres en Tunisie », Journal Officiel, n $104^{19}$.

MOADA, Mohammed (1986) : (en arabe) Harakat at-tarjama fi tounis, 1840-1955. (Le mouvement de traduction en Tunisie 1840-1955). Tunis-Tripoli, Maison Arabe du Livre.

MOUCHARRAFIEH, Madiha (1984) : (en arabe) Al-muassasat at-taqafya fi-l-ayala at-tounisya fi-n-nisf al-awwel min al-qarn aitasi' 'achar (1830-/855), (Les Institutions culturelles dans la Régence de Tunis à la 
première moitié du XIXe siècle (I830-I855). Tapuscrit. n T 3125. Tunis, Faculté des Sciences Humaines et Sociales.

MOUCHARRAFIEH, Madiha (1990) : (en arabe) Al-madaris al-horra fi-l-bUad at-tou-nisya 1855/56-1885/86, [Les écoles libres en Tunisie (1855/56-1855/1886)], Mémoire de DEA, tapuscrit. Tunis, Faculté des Sciences Humaines et Sociales.

PIOLET, Jean Baptiste (1902) : Les Missions catholiques françaises au XIX siècle. Tome 5. Paris, Armand Colin.

PIOLET, Jean Baptiste (1902) : Les Missions catholiques en Orient et en Afrique du Nord. Tome 1 et 4. Paris, Armand Colin.

SAKGI, Habib (1973) : La politique de l'enseignement en Tunisie ci la veille de la première guerre mondiale 1881-1914. Mémoire de DEA, Tapuscrit T. 2400. Tunis. Faculté des Sciences Humaines et Sociales.

TOURNIER, Jules (1913) : Le cardinal Lavigerie et son action politique 1863-1892). Paris, Perrin et Cie.

\section{NOTES}

1. Il semblerait que l'afflux des étrangers en Tunisie ne soit pas dû à une démonstration de force, comme le pense Chater, mais plutôt à la fin de la course, imposée à la Berbérie, par les grandes puissances de l'époque, lors de la conférence d'Aix, en 1819.

2. Les Maltais y étaient poussés par la pauvreté, les Siciliens par la promesse de l'embauche et par la proximité de la Tunisie, la Sicile n'étant qu'à $140 \mathrm{~km}$ des côtes tunisiennes.

3. L'école fondée en 1830 par les Anglais rue Portofarina (avec deux parties une pour les garçons et une pour les filles) était présidée par le Dr. Davis. Certains, comme Louis Machuel, pensent qu'elle a été fermée plus tard pour des raisons économiques. En réalité, les Anglais majoritairement protestants - voyant qu'ils avaient été définitivement battus sur ce terrain par les Italiens et les Français et considérant leurs sujets maltais (catholiques), plus proches des Italiens, n'avaient pas voulu investir davantage. En outre, l'Angleterre, ayant acquis l'île de Chypre (en 1878), avait voulu faire un geste en direction de la France en lui laissant les mains libres en Tunisie. Il n'était désormais plus question de s'investir en Tunisie.

4. Après les événements de 1822, 1830, 1848, etc., certains avaient pensé que la libéralisation de l'enseignement permettrait la diffusion du savoir et serait en mesure d'éradiquer la violence en cultivant l'esprit de tolérance et en affermissant les bases du civisme.

5. Ce transfert du pouvoir s'est opéré lors du règne de Hassine Bey (1824-1835).

6. Fondatrice, à Gaillac, en 1832. de la congrégation des Sœurs de Saint-Joseph

7. Nous verrons que le nombre de Français qui auraient profité de cet enseignement était plus qu'insignifiant.

8. Encore en usage de nos jours en Tunisie pour l'essentiel.

9. Auteur lui-même d'ouvrages sur l'enseignement dans la Régence de Tunis. Voir notre bibliographie.

10. Qui continue jusqu'à ce jour.

11. Cf. Les écrivains francophones de Tunisie, italiens, juifs et musulmans.

12. Documents des Archives de la République Tunisienne, ceux des Pères Blancs de Rome, ceux des Frères de Saint Joseph, ceux des Frères des Écoles Chrétiennes (rue de la Casbah), thèses et travaux d'historiens, ainsi que les travaux de recherche sur les institutions d'enseignement en Tunisie avant le protectorat.

13. Pour en déterminer le nombre et les origines, v. H. Dunant 1858 et J. Ganiage 1955.

14. À l'exception toutefois du secrétaire de l'évêque, le père Anselme, qui était français. 
15. Marcel Gandophe (1923 : 241) nous dit, pour justifier le désengagement de l'Angleterre, que les Italiens et les Maltais considéraient l'école de la rue de l'Église, parce qu'elle était occupée principalement par ces deux communautés « comme leur appartenant ; aussi l'Angleterre, depuis cette création n'a plus tenté aucun effort pour établir des institutions scolaires... ».

16. Ce qui a valu au Père Bourgade de subir la persécution des prêtres capucins italiens et à leur tête l'évêque Sutter.

17. Le Pacte Fondamental met les étrangers sous la protection des consuls de France ou d'Angleterre et donne à ces étrangers, entre autres avantages, le droit de posséder des biens immeubles en Tunisie.

18. Mis à part quelques allusions peu crédibles sur les méthodes d'enseignement, du genre de ceux de Pierre Louis Machuel (1889), qui était plutôt porté sur les exagérations emphatiques, affirmant qu'on suivait presque le même programme qu'en France.

19. Paul Melon était délégué de l'Alliance Française en Tunisie et Tripoli.

\section{RÉSUMÉS}

Notre propos est de faire le point sur les conditions, les buts et surtout le statut de la langue française dans l'enseignement dispensé en Tunisie par les écoles libres (qu'elles soient françaises ou tunisiennes, autochtones ou relevant de l'Alliance Israélite Universelle) avant l'installation du Protectorat, c'est-à-dire de 1830 à 1883.

Our goal is to appraise the context, purposes, and especially the status of French as a language taught in private schools in Tunisia - whether they are French or Tunisian, local or related to the Universal Israelite Alliance - before the Protectorat, i.e. from 1830 to 1883.

\section{INDEX}

Mots-clés : Tunisie, protectorat, enseignement du français, écoles libres, Alliance israélite universelle

Keywords : Tunisia, protectorat, french teaching, private schools, Universal Israelite Alliance

\section{AUTEUR}

\section{ABDERRAZAK BANNOUR}

Université de Tunis 